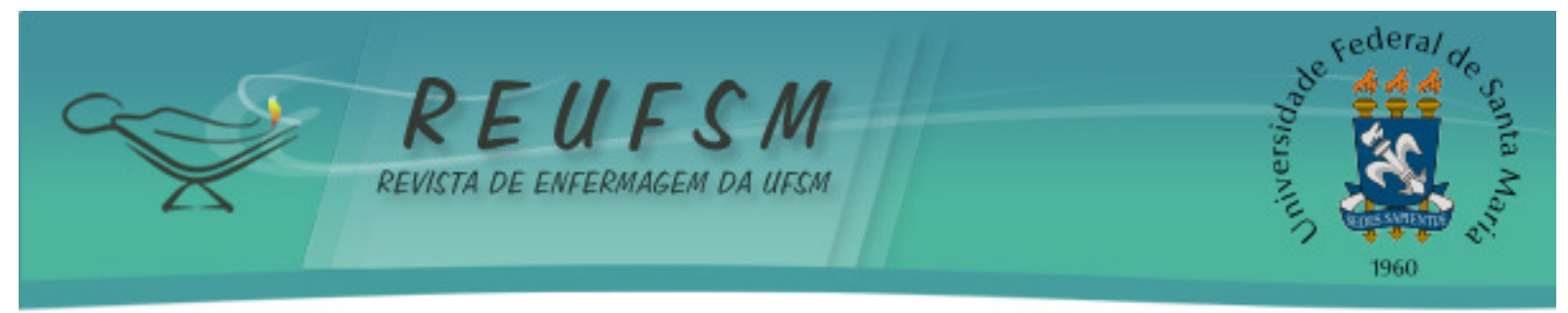

ARTIGO ORIGINAL

\title{
PERCEPÇÃO DOS USUÁRIOS ACERCA DO ATENDIMENTO NO CENTRO DE ATENÇÃO PSICOSSOCIAL III
}

\author{
PERCEPTION OF USERS ABOUT THE CARE AT THE PSYCHOSOCIAL CARE \\ CENTER III
}

\section{PERCEPCIÓN DE LOS USUARIOS SOBRE LA ATENCIÓN EN EL CENTRO DE ATENCIÓN PSICOSOCIAL III}

Doi: $10.5902 / 2179769225942$

\author{
Francisco de Sales Clementino ${ }^{1}$ \\ Flávia Gomes Silva ${ }^{2}$ \\ Francisco Arnoldo Nunes de Miranda ${ }^{3}$ \\ Débora Taynã Gomes Queiróz ${ }^{4}$ \\ João Mário Pessoa Júnior ${ }^{5}$ \\ Emanuella de Castro Marcolino ${ }^{6}$
}

RESUMO: Objetivo: analisar a percepção dos usuários atendidos no CAPS III sobre o atendimento prestado pelos profissionais de saúde neste serviço. Método: pesquisa qualitativa, descritiva e exploratória, realizada entre novembro de 2015 e maio de 2016 no município de Campina Grande, Paraíba. Foi utilizado um questionário autoplicado junto a 47 usuários atendidos num CAPS III. Os resultados foram discutidos à luz da Análise de Conteúdo Temática. Resultados: Obteve-se duas categorias temáticas, a saber: facilidades e potencialidades; e barreiras a vencer: geográfica, organizacional, econômica e de estrutura. Observou-se que a satisfação dos usuários relaciona-se ao atendimento, assistência e escuta qualificada prestada pelos profissionais de saúde do CAPS, mesmo frente a uma estrutura física precária, o dimensionamento inadequado dos profissionais e a falta de medicamentos. Conclusão: Os usuários do CAPS III inferiram satisfação com o acolhimento prestado pelos profissionais de saúde; todavia, também apresentaram entraves do próprio serviço em termos organizacionais e estruturais.

Descritores: Saúde mental; Serviço de saúde; Avaliação em saúde.

\footnotetext{
${ }^{1}$ Enfermeiro e Professor da Graduação em Enfermagem da Universidade Federal de Campina Grande, Doutor em Enfermagem pela Universidade Federal do Rio Grande do Norte, Universidade Federal de Campina Grande, Campina Grande, Paraíba, Brasil, fclementino67@yahoo.com.br

${ }^{2}$ Enfermeira, Graduação em Enfermagem pela Universidade Federal de Campina Grande, Universidade Federal de Campina Grande, Campina Grande, Paraíba, Brasil, flavianag12@gmail.com

${ }^{3}$ Enfermeiro e Professor do Departamento de Enfermagem da Universidade Federal do Rio Grande do Norte, Doutor em Pós-doutorando em Enfermagem na Universidade de Évora, Universidade Federal do Rio Grande do Norte, Natal, Rio Grande do Norte, Brasil, farnoldo@gmail.com

${ }^{4}$ Enfermeira, Mestranda em Enfermagem pelo programa associado em pós-graduação pela Universidade de Pernambuco e Universidade Estadual da Paraíba, Universidade de Pernambuco e Universidade Estadual da Paraíba, Campina Grande, Paraíba, Brasil, debora-tayna@ hotmail.com

${ }^{5}$ Enfermeiro e Professor da Graduação em Medicina da Universidade Federal Rural do Semi-Árido, Doutor em Enfermagem pela Universidade Federal do Rio Grande do Norte, Universidade Federal Rural do Semi-Árido, Mossoró, Rio Grande do Norte, Brasil, joaomariopessoa@gmail.com

${ }^{6}$ Enfermeira e Professora da Graduação em Enfermagem da Faculdade de Ciências Médicas, Doutoranda em Enfermagem pela Universidade Federal do Rio Grande do Norte, Faculdade de Ciências Médicas, Campina Grande, Paraíba, Brasil, emanuella.de.castro@gmail.com
} 


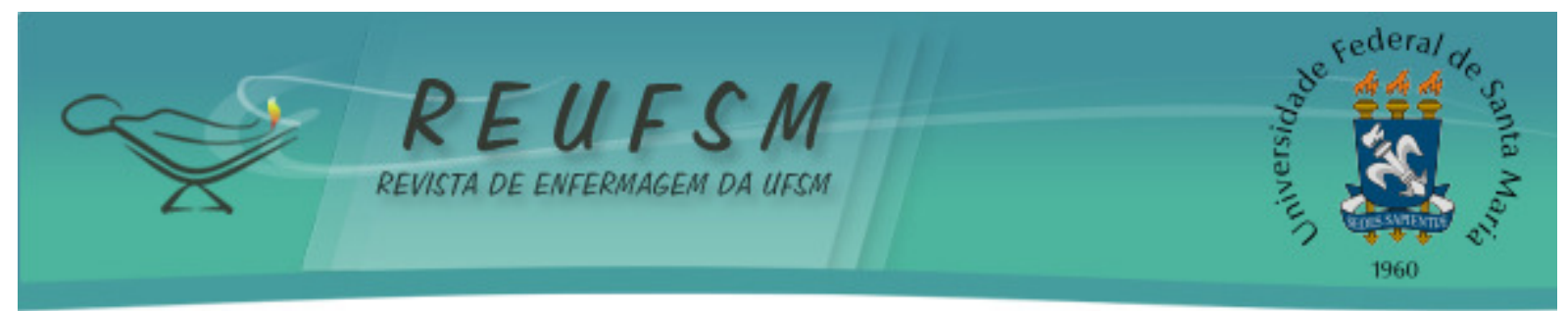

ABSTRACT: Aim: to analyze the perception of users attended at the CAPS III about the care provided by health professionals in this service. Method: qualitative, descriptive and exploratory research, carried out from November 2015 to May 2016 in the city of Campina Grande, Paraíba. A self-administered questionnaire was used with 47 users attending a CAPS III. The results were discussed in light of the Thematic Content Analysis. Results: Two thematic categories were obtained: facilities and potentialities; And barriers to overcome: geographical, organizational, economic and structure. It was observed that the satisfaction of the users is related to the care, assistance and qualified listening provided by the health professionals of the CAPS, even in the face of a precarious physical structure, inadequate professional dimensioning and lack of medication. Conclusion: CAPS III users claimed to be satisfied with the care provided by health professionals; However, they also presented organizational and structural obstacles to the service itself.

Descriptors: Mental health; Health Service; Health assessment.

RESUMEN: Objetivo: analizar la percepción de los usuarios atendidos en el CAPS III sobre la atención prestada por los profesionales de salud en este servicio. Método: investigación cualitativa, descriptiva y exploratoria, realizada entre noviembre de 2015 y mayo de 2016, en el municipio de Campina Grande, Paraíba. Se utilizó un cuestionario autoplicado con 47 usuarios atendidos en el CAPS III. Los resultados fueron discutidos con base en análisis de contenido temático. Resultados: Se obtuve dos categorías temáticas, a saber: facilidades y potencialidades; y dificultades a vencer: geográfica, organizacional, económica y de estructura. Se observó que la satisfacción de los usuarios está relacionada con la atención, asistencia y escucha calificada prestada por los profesionales de salud del CAPS, aunque delante de una estructura física precaria, del dimensionamiento inadecuado de los profesionales y de la falta de medicamentos. Conclusión: Los usuarios del CAPS III evidenciaron satisfacción con la acogida prestada por los profesionales de salud; sin embargo, también identificaron obstáculos en el servicio en términos organizativos y estructurales.

Descriptores: Salud mental; Servicio de salud; Evaluación en salud.

\section{INTRODUÇÃO}

Historicamente, no ano de 2001, o Brasil alcançou um importante passo relacionado a assistência em saúde às pessoas com transtorno mental com a criação da Lei $\mathrm{n}^{\circ}$ 10.216, conhecida como a Lei da Reforma Psiquiátrica (RP), a qual sustenta a eliminação gradativa dos manicômios e a substituição dos mesmos por novas modalidades de atendimento. As novas proposições de cuidado em saúde mental tiram do foco a doença e passam a enfatizar o sujeito, valorizando de sobremaneira a interação entre profissional e usuário em seu processo de tratamento e reinserção na sociedade. ${ }^{1}$

Com vistas a um serviço comunitário, interdisciplinar, intersetorial, emergem os serviços substitutivos e intermediários de atenção à saúde mental. Dentre estes, destaca-se os Centros de Atenção Psicossocial (CAPS), Hospitais-Dia, Serviços Residenciais Terapêuticos, Centros de Convivência e Cultura, Consultórios de Rua, entre outros. Ademais, o Ministério 


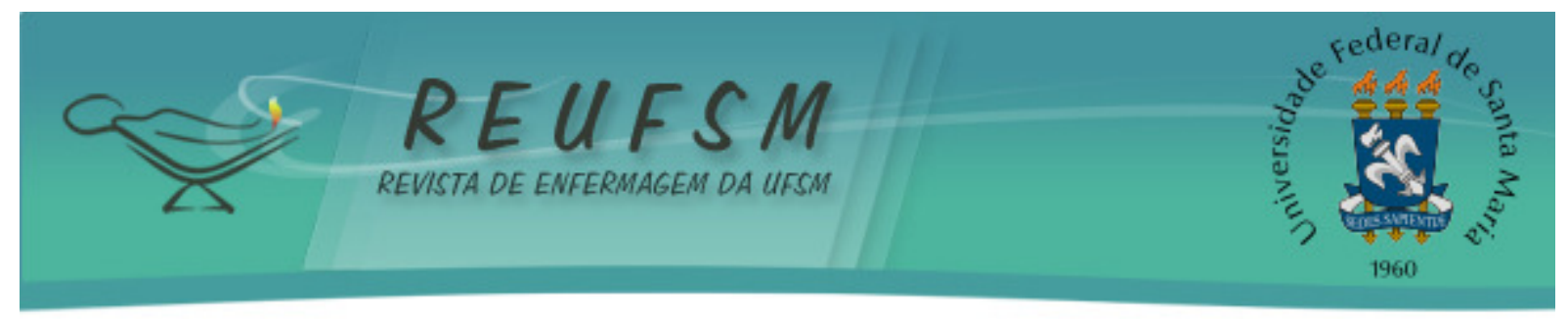

da Saúde (MS) institui a Rede de Atenção Psicossocial (RAPS), no ano de 2011, por meio da Portaria $n^{\circ} 3.088$. A RAPS possui como objetivo ampliar e promover o acesso da população aos serviços especializados e pontos de atenção à saúde no território, na busca de qualificar o cuidado e acompanhamento contínuo, além de atenção às urgências. ${ }^{2}$

No contexto da RAPS, os CAPS configuram-se como dispositivos estratégicos no atendimento de atenção diária ao usuário com transtorno mental, promovendo sua inserção social. Além disso, regula a porta de entrada da rede de assistência no apoio das ações de saúde mental na rede básica. Considera-se, portanto, como um importante benefício da RP, uma vez que o tratamento acontece em ambientes abertos, humanizados e mais próximo do convívio comunitário. ${ }^{3}$

Mesmo diante dos avanços obtidos com ampliação da cobertura de CAPS e a gradativa implementação da RAPS entre os municípios brasileiros observam-se problemas estruturais como a escassez de financiamento na área de saúde mental, falta de recursos humanos qualificados, entre outros. Tais fatores influenciam diretamente na assistência em saúde mental ofertada e na própria percepção dos usuários sobre a qualidade dessa rede. ${ }^{4}$

Partindo desse cenário, torna-se necessário analisar os aspectos que influenciam o processo de atenção à saúde mental no nível qualitativo a partir do grupo de usuários que utilizam esse serviço. ${ }^{1-2}$ Diante do exposto, têm-se os seguintes questionamentos: qual a percepção dos usuários sobre o atendimento oferecido pelos profissionais do CAPS III? Quais as dificuldades em relação ao acesso às ações de saúde? Eles reconhecem o CAPS III como promotor da reinserção social? Assim, o presente estudo objetiva analisar a percepção de usuários atendidos no CAPS III sobre o atendimento prestado pelos profissionais de saúde neste serviço.

\section{MÉTODO}

Estudo descritivo e exploratório, com abordagem qualitativa, realizado entre novembro de 2015 e maio de 2016, no Centro de Atenção Psicossocial III (CAPS III), do município de Campina Grande, Paraíba.

A amostra foi composta por 47 usuários, tendo como critérios de inclusão: idade igual ou superior a 18 anos; registro de admissão no CAPS III e ser ativos no atendimento. E, de exclusão: estar comprometido psiquicamente, em crise ou em uso de medicamentos que impossibilitassem a resposta ao instrumento do estudo, correspondendo um total de cinco usuários. 


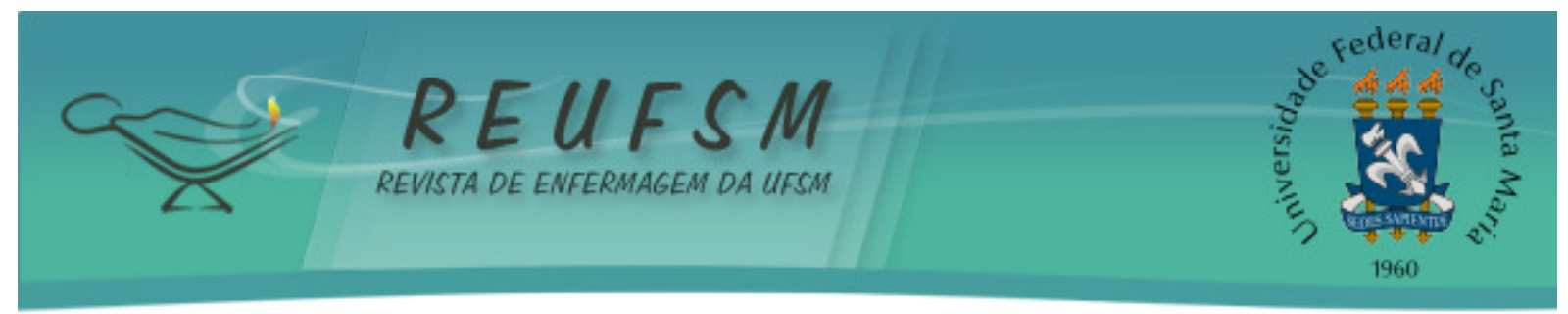

A escolha dos participantes e o local da pesquisa combinaram com os critérios de conveniência e intencionalidade. Para atender ao primeiro critério foi considerado o período disponível para a coleta de dados, bem como o acesso dos pesquisadores junto aos participantes. Para definir o quantitativo de usuários, agregou-se o critério de intencionalidade e o critério de saturação teórica da amostra, quando as informações contidas nas entrevistas tornam-se redundantes. ${ }^{5}$

A escolha do CAPS III se deu pelo tipo assistência oferecida, dispondo de uma equipe multiprofissional, além de ser considerado serviço regulador no contexto da urgência na RAPS com três modalidades de atendimento: intensiva, semi-intensiva e não intensiva, funcionando 24 horas, inclusive feriados e finais de semana.

A coleta dos dados foi realizada com um formulário elaborado para o estudo CAPSUL (Avaliação dos CAPS da Região Sul do Brasil) ${ }^{6}$, composto por duas partes, a saber: a primeira referente à caracterização sociodemográfica dos participantes do estudo. A segunda parte compreendeu questões abertas orientadoras, que serviram para conhecer a percepção dos participantes quanto ao nível de satisfação sobre o CAPS III. Ao todo, foram aplicados 47 questionários individuais, preenchido pelo pesquisador, antes ou após o atendimento no CAPS, com duração média de 30 minutos cada instrumento, em salas do próprio serviço garantindo a privacidade e a confidencialidade das informações.

Para a análise dos dados utilizou-se a técnica de codificação dos conteúdos das falas, composta pelas fases de pré-análise, codificação, tratamento dos resultados, inferência e interpretação, segundo Bardin. ${ }^{7}$ A pré-análise compreendeu a fase de organização do conteúdo com o objetivo de sistematizar as ideias iniciais; a segunda fase de codificação, que representou a análise propriamente dita, consistiu em decodificação, decomposição ou enumeração dos dados; após análise os resultados obtidos foram tratados de maneira a tornarem significativos, e a partir de então foi possível propor inferências e adiantar interpretações. ${ }^{7}$ Os dados numéricos foram analisados através da estatística descritiva (frequências absoluta e percentual).

Atendendo à Resolução No 466/12 do Conselho Nacional de Saúde do Brasil, que dispõe sobre pesquisas envolvendo seres humanos, o estudo foi aprovado pelo Comitê de Ética em Pesquisa da Universidade Federal do Rio Grande do Norte (UFRN), recebendo Parecer favorável sob Protocolo o No 719.435/14, em 30/05/2014. Para preservar o anonimato 


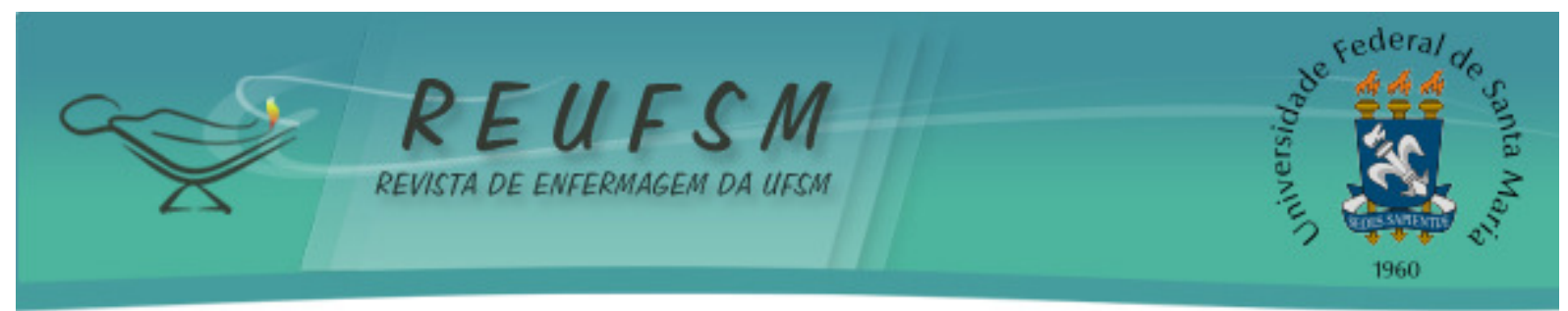

dos participantes do estudo, os mesmos foram cognominados com alcunhas de atletas que participaram das "Olimpíadas de 2016”.

\section{RESULTADOS E DISCUSSÃO}

Quanto às características sociodemográficas dos 47 usuários, 33 (70,21\%) eram do sexo feminino e $14(29,79 \%)$ do sexo masculino. A faixa etária predominante entre 27 usuários $(57,44 \%)$ abarcou os adultos de 36 a 60 anos, em segundo patamar os adultos jovens 12 (25,53\%), em seguida os adolescentes até 20 anos três $(6,39 \%)$, mesmo percentuais dos que não souberam e por fim os idosos dois $(4,25 \%)$. Em relação ao estado civil, 26 (25,53\%) casado ou com companheiro, viúvo/viúva cinco (10,63\%), divorciado três (6,39\%), e separado um $(2,13 \%)$.

No que se refere aos aspectos econômicos, a principal fonte de renda para 13 usuários $(27,65 \%)$ se baseava no auxílio-doença, para dez $(21,28 \%)$ renda familiar, para sete usuários $(14,89 \%)$ outras formas, para seis $(12,76 \%)$ aposentadoria, para quatro $(8,51 \%)$ renda do cônjuge/companheiro, para três $(6,39 \%)$ emprego, para três $\left(6,69^{*} \%\right)$ nenhuma fonte, para um (2,13\%) seguro-desemprego. A renda mensal de 24 usuários $(51,07 \%)$ encontrava-se em torno de um a dois salários mínimos, 13 (27,65\%) menos de um salário mínimo, nove $(19,15 \%)$ não sabiam a renda, um $(2,13 \%)$ acima de dois salários mínimos.

A partir da análise dos dados emergiram duas categorias. A primeira categoria evidenciou as "Facilidades e potencialidades" frente ao atendimento recebido no CAPS III. A segunda categoria, denominada "Barreiras a vencer": geográfica, organizacional, econômica e de estrutura, enfatiza as dificuldades enfrentadas pelos usuários frente à acessibilidade às ações de saúde.

\section{Facilidades e potencialidades}

Os resultados apontam a satisfação dos usuários com relação à qualidade do atendimento oferecido pelo CAPS III, deixando transparecer o contentamento em relação ao acolhimento recebido durante todo o seu processo de adoecimento. Neste sentido, ao questionar o usuário, obteve-se como respostas:

Gosto do atendimento, pois sempre quando precisei me trataram bem, quando estamos com crise eles tratam com calma. (Jaqueline Ferreira)

"Gosto do CAPS por ser bom, foi aqui que encontrei a segunda casa, onde melhorei. Sou bem atendida". (Sheila Castro)

"Porque fui bem acolhida, tem bons profissionais". (Flávia Saraiva) 


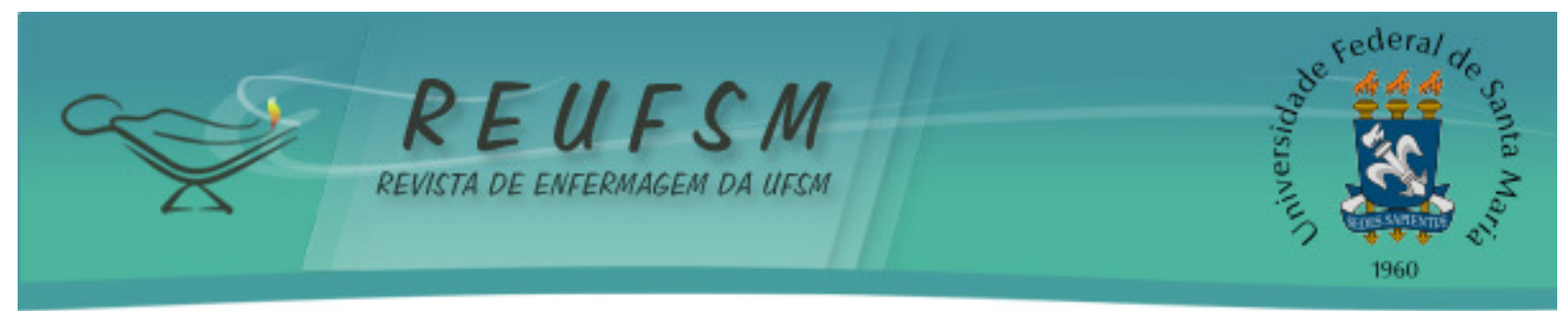

Os relatos apontaram que os usuários estavam satisfeitos em relação ao tratamento, à ajuda recebida e acolhida da equipe, além das condições físicas e conforto do serviço. Esse achado também foi encontrado em outro estudo sobre satisfação de usuários em CAPS ad. ${ }^{2}$

Na saúde mental, a escuta, o acolhimento e o vínculo caracterizam-se como ações preponderantes para as intervenções, consideradas tecnologias estratégicas para o cuidado no território, permitindo, dessa forma, uma "intimidade terapêutica" no sentido de o trabalhador estar aberto à escuta das necessidades de saúde do usuário, em uma postura mais acolhedora. ${ }^{8}$ O termo acolher define-se como a maneira de receber ou de ser recebido, abrigo gratuito e hospitalidade, saber ouvir com atenção, dar crédito, admitir, aceitar. Trata-se de uma oportunidade de estabelecer relações produtoras de vínculo, através da escuta solidária e do comprometimento com a trajetória do usuário no serviço. ${ }^{8-9}$

Observou-se a partir dos resultados obtidos, uma relação empática que embasou a responsabilização profissional e a formação de vínculo entre a equipe, usuário e familiar. Contudo, pesquisa realizada com o intuito de avaliar o processo de acolhimento em Saúde Mental na região centro-oeste do município de São Paulo, apontou a vinculação da triagem com o acolhimento, sem alterar significativamente as práticas, não possibilitando o espaço da fala, não reorganizando a rede de cuidados e não investindo em vínculos com os usuários. ${ }^{10}$

Observa-se que a oferta diversificada de oficinas terapêuticas no CAPS constitui uma estratégia bem aceita entre os grupos de usuários, pois reconhece suas necessidades individuais e valorizam as aptidões para o desenvolvimento de atividades manuais ou cognitivas, como as oficinas de artesanato, escrita, música, jornal, entre outras. Além disso, a escuta ativa possibilita que o usuário compartilhe e faça reflexões sobre suas fantasias, medos, emoções:

Gosto das oficinas, pois trabalha a mente. (Flávia Saraiva)

Da oficina terapêutica de terça-feira. Gosto da psicóloga que atende a gente, procura saber como a gente está indo. (Rafaela Silva)

A análise das percepções dos usuários sobre as oficinas terapêuticas permite apreender diferentes ideias, tais como: as oficinas precisam favorecer a expressão dos usuários e a busca de autonomia, cidadania e novas relações entre sujeitos e grupos, sendo instrumentos clínicos e políticos. ${ }^{11}$ As oficinas terapêuticas podem ser consideradas como um espaço promotor de potencialidades e habilidades para a expressão da subjetividade dos usuários assistidos em CAPS. Pesquisa realizada nos CAPS com os profissionais de saúde na cidade de Pelotas, RS, observou que 


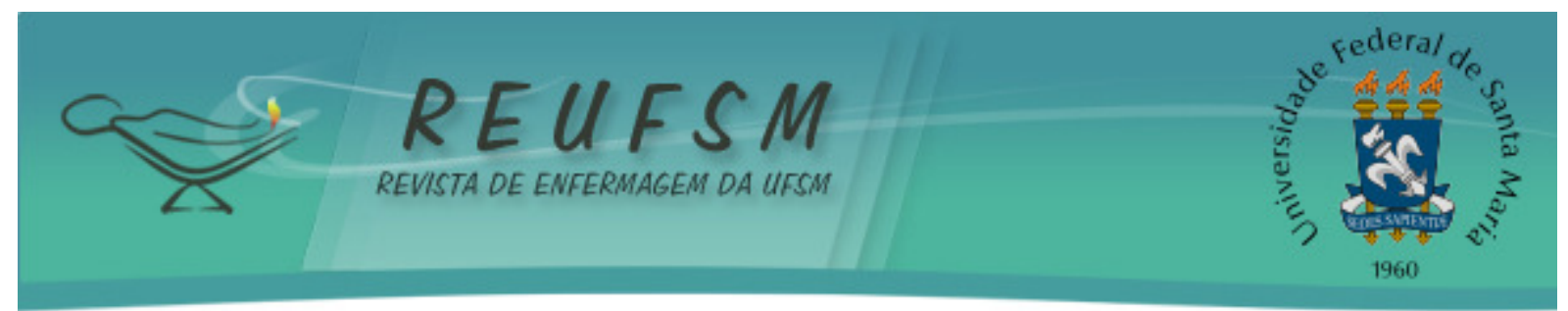

a equipe reconhece a importância das oficinas terapêuticas para o usuário dos CAPS por possibilitar aos envolvidos a participação ativa no seu processo de reabilitação psicológica e social. ${ }^{11}$

Outro estudo, realizado em um CAPSi localizado em Santa Catarina, com familiares de adolescentes atendidos no serviço de saúde supracitado, observou-se nas falas dos familiares, percepções acerca das oficinas terapêuticas, relacionadas à importância para o tratamento de seu familiar. Entende-se que as tecnologias de cuidado que contribuem para a melhora da convivência social e familiar, a redução dos danos associados ao consumo de drogas e a estabilidade e diminuição das crises. ${ }^{12}$

Os usuários expressaram sentimentos de empatia, aceitação, relaxamento, respeito às diferenças e segurança em relação à equipe do CAPS. Além disto, os usuários reconhecem o CAPS III como espaço de motivação, por trazer conhecimentos e ampliar possibilidades de cuidar da sua própria saúde:

Porque seria mais um relaxamento para mim, mais um aprendizado, porque você aprende a aceitar as adversidades das pessoas, as diferenças de cada um (Rafaela Silva); porque, às vezes, recebe as pessoas e dá mais atenção do que no hospital geral. (Isaquias Queiroz)

Porque o local é bom. Cheguei quase louca e estou bem melhor. (Ingrid Oliveira)

A partir do convívio e/ou contato com outras pessoas que enfrentam os desafios no lidar com os transtornos mentais, os usuários acolhidos no CAPS ressignificam suas experiências de vida, aprendem a lidar com a diversidade e, de alguma forma, buscam superar dificuldades advindas pós-adoecimento. Nesse sentido, torna-se também necessário fortalecer a rede de apoio (como família, amigos e comunidade no geral) em torno dos que recebem as ações de cuidados em saúde mental, seja oferecendo-lhes esclarecimentos e compartilhando responsabilidades, seja destinando-lhes o suporte adequado. ${ }^{13}$

Outro aspecto de satisfação citado por uma usuária do CAPS diz respeito às estratégias de reinserção no mercado de trabalho, na medida que reconhece o serviço um meio complacente para oportunizar tal ferramenta:

Ter mais oportunidades de outras coisas (cursos) de costura, aprender a trabalhar em indústria e ganhar o seu dinheiro. Aprender uma profissão. (Rafaela Silva)

A fala da usuária aponta para o fortalecimento da ideia de geração de renda no contexto dos serviços e a necessidade de maiores investimentos na formação profissional. Entende-se que 


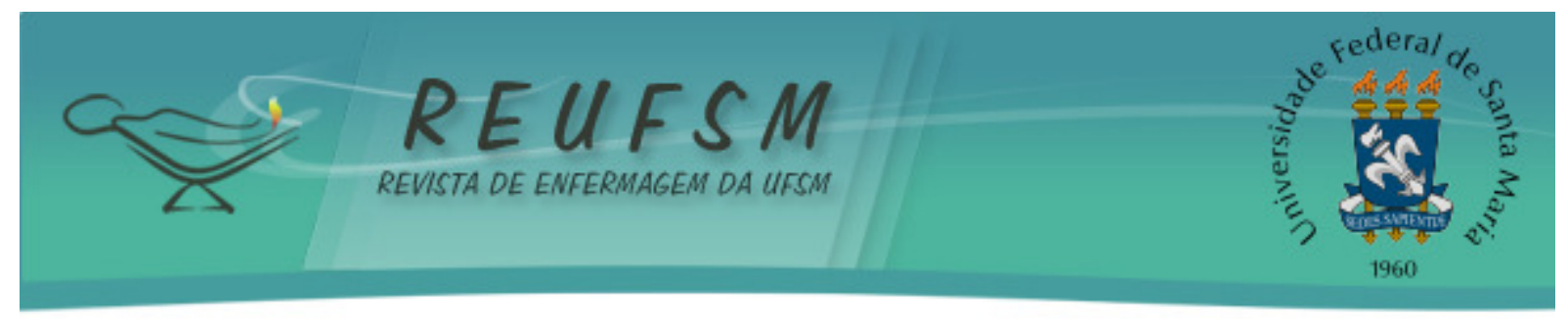

o trabalho como instrumento fundamental para promover a reinserção do pessoal com transtorno mental na sociedade através da geração de renda. Desse modo, as intervenções em saúde mental necessitam promover novas possibilidades de modificar e qualificar as condições e modos de vida dos usuários, orientando-se pela produção de saúde e de vida. ${ }^{14}$

De um lado, a ideia da inclusão pelo trabalho se coloca não apenas como um dispositivo de políticas públicas voltadas à criação de um novo modelo assistencial em saúde mental, mas enquanto um processo vivo constituinte de um novo campo social, de afirmação e busca de direitos. ${ }^{15}$ Do outro, o CAPS, embora percebido como um local de acolhimento, seguro para os usuários, estes são vistos com preconceito por frequentarem um local de assistência psicológica e não estão inseridos no mercado de trabalho, fracassando na inserção social de maneira plena, requerendo o extrapolar para outras dimensões da vida social. ${ }^{16}$

No contexto atual de capitalismo, as pessoas com transtornos mentais ou mesmo que apresentam algum tipo de deficiência física são excluídas dos meios de produção e passam a condição objetificada de "doente mental” e, por conseguinte, excluídos do mercado do trabalho. O estímulo a geração de renda entre os serviços de saúde mental fortalece a autonomia e empoderamento do usuário na medida em que recebe um incentivo e recompensa pelo trabalho realizado. ${ }^{17-18}$

Assim, a ênfase na promoção da saúde não se restringe apenas as ações e práticas voltadas ao tratamento dos transtornos mentais ou tampouco a resolução da crise psíquica, ainda no modelo hospitalocêntrico, mas avance em direção à cidadania na medida em que reconhece a multimensionalidade do processo saúde-doença mental e considera o sujeito no seu contexto de vida. ${ }^{13-15}$

\section{Barreiras a vencer: geográfica, organizacional, econômica e de estrutura}

Os usuários relatam, no cotidiano do CAPS III, presença de barreiras relacionadas à própria organização do serviço de saúde. Dentre estas, se destaca a localização geográfica, que, na maioria das vezes, ocasiona uma barreira econômica, uma vez que necessita do recurso financeiro para se deslocar até o serviço. Além disso, as crescentes interferências ocasionadas pelas barreiras organizacionais e de estrutura física e humana. 


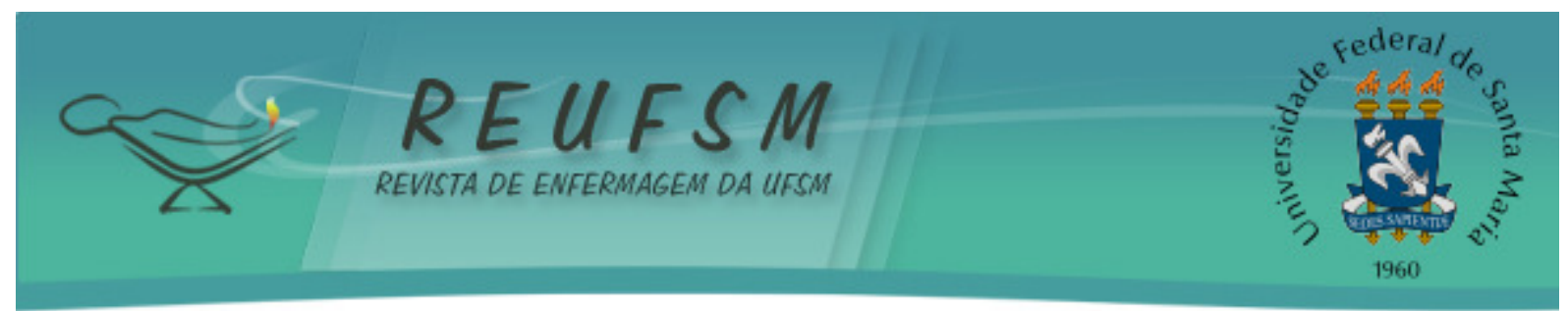

Em relação à barreira geográfica, os usuários expressaram que o fator geográfico é relevante para garantir o acesso dos usuários aos serviços de saúde:

O transporte que não tenho, é muito cansativo. Venho a pé para o CAPS III. Não tenho ajuda de custo para o transporte. (Rafaela Silva)

Podia ser melhor, se fossem para a STTP [Superintendência de trânsito e transportes] pra conseguir o cartão, pra gente vim pra cá. (Marina Canetti).

Mesmo satisfeitos com o atendimento, os usuários indicaram algumas fragilidades. Trata-se de elementos que reportam à conveniência do horário de funcionamento dos serviços, tempo de espera para consultas, insumos, medicamentos, dentre outros:

A medicação que falta, e não posso comprar, se tivesse era melhor! (Andreia Bandeira)

Disponibilidade da medicação, às vezes falta. (Maria Portela)

Pesquisadores ${ }^{18}$ encontraram resultados semelhantes no tocante à insatisfação quanto à falta de medicação, à insuficiência de recursos humanos e às salas de atendimento inadequadas. Tal situação contribui efetivamente para o acesso negativo aos serviços de saúde mental e na diminuição da qualidade do serviço prestado.

Além disso, foram identificados problemas como a falta de comunicação e informação sobre o atendimento ofertado e a elevada demanda de usuários no serviço, características que impactam no acesso e qualidade do atendimento:

Nas consultas, é descontrolado. Falta de informação dos profissionais a respeito do atendimento, horário. (Rodrigo Pessoa)

Às vezes não entendo nada nas consultas, é muita gente e muito barulho, só tem um médico para atender. (Maria Portela)

A comunicação e o acesso à informação são elementos fundamentais para o acolhimento em saúde, além disso, fortalecem o vínculo do usuário junto ao serviço. Evidencia-se, em alguns momentos, devido a sobrecarga de atividades e/ou demanda excessiva de usuários, falha de comunicação por parte dos profissionais, levando a conflitos. Entende-se que o ato de esclarecer acerca das atividades e procedimentos a serem realizados minimizam tal questão, contribuindo para a realização do cuidado humanizado. ${ }^{19}$

As falas destacaram ainda a necessidade de maior estruturação do serviço, no que diz respeito à contratação de funcionários, melhoria da estrutura física, alimentação, dentre outras. Como se pode perceber nos relatos a seguir: 


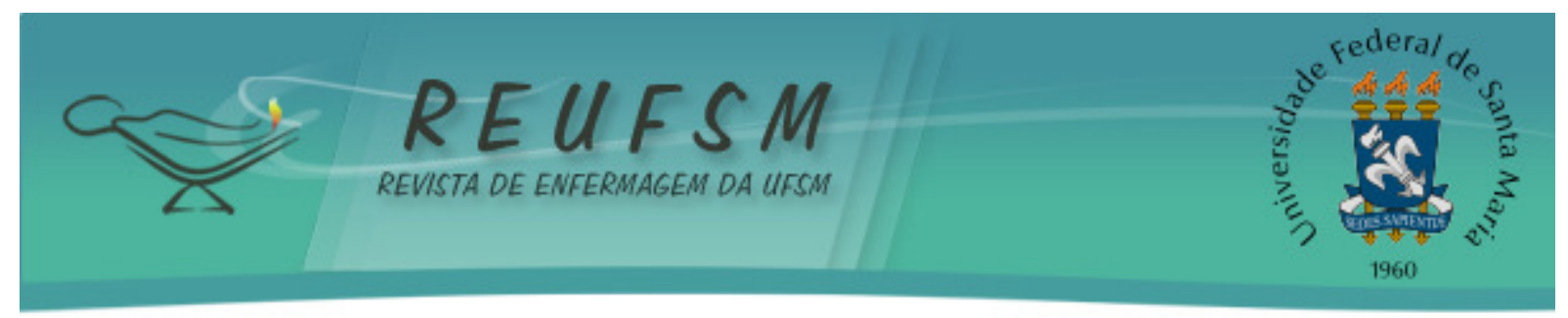

Ter mais funcionários, não tem muito, e deveriam buscar melhoras. (Ana Sátila)

O banheiro deveria ter mais higiene, ser separado, ter o do homem e o da mulher. (Lara Teixeira)

Alimentação é muito precária. Ter limpeza no CAPS. (Flávia Saraiva)

No concernente à gestão e organização dos serviços de saúde mental, os aspectos estruturais exercem papel importante, com influência direta na operacionalização das ações e cuidados entre os serviços, carecem desse modo, de maiores investimentos com vistas a minimizar as fragilidades atribuídas pelos sujeitos do estudo.

Os resultados encontrados no presente estudo são equivalentes aos do estudo realizado no CAPS III do município de Caicó/RN, no qual mais de $60 \%$ dos sujeitos entrevistados relataram uma avaliação negativa quanto aos recursos humanos disponibilizados e $100 \%$ apontaram que os recursos financeiros e materiais do CAPS são insuficientes. ${ }^{20}$ Outro estudo que avaliou o perfil dos usuários do Ambulatório de Saúde Mental e do Centro de Atenção Psicossocial de Lorena-SP identificou que a precariedade da estrutura física e o número insuficiente de profissionais afeta de maneira negativa a assistência ofertada. ${ }^{19} \mathrm{O}$ espaço físico oportuniza a liberdade e autonomia do usuário, pois favorece a participação e a interação do mesmo com a equipe e com os colegas, além de ser uma oportunidade para movimentar-se com leveza produzindo prazer e bem estar. ${ }^{3}$

Os trabalhadores relataram ainda que existe uma forte instabilidade profissional devido às mudanças de governo, o que acarreta uma dificuldade na construção do vinculo e confiança entre profissional e paciente.

\section{CONCLUSÃO}

Os usuários evidenciaram satisfação em relação ao atendimento e ao acolhimento, incluindo à escuta qualificada e o seguimento, prestados pelos profissionais no CAPS III, além de compreenderem o serviço como ambiente promotor de reinserção social a medida que têm a oportunidade de capacitação e reintegração no mercado de trabalho; desse modo, o ambiente institucional e a equipe contribuem para o bem-estar e acolhimento. Por outro lado, os usuários analisam o acesso ao serviço de saúde mental permeados de entraves, os quais compreendem: acesso geográfico difícil quanto a distância da residência em relação ao serviço, organização 


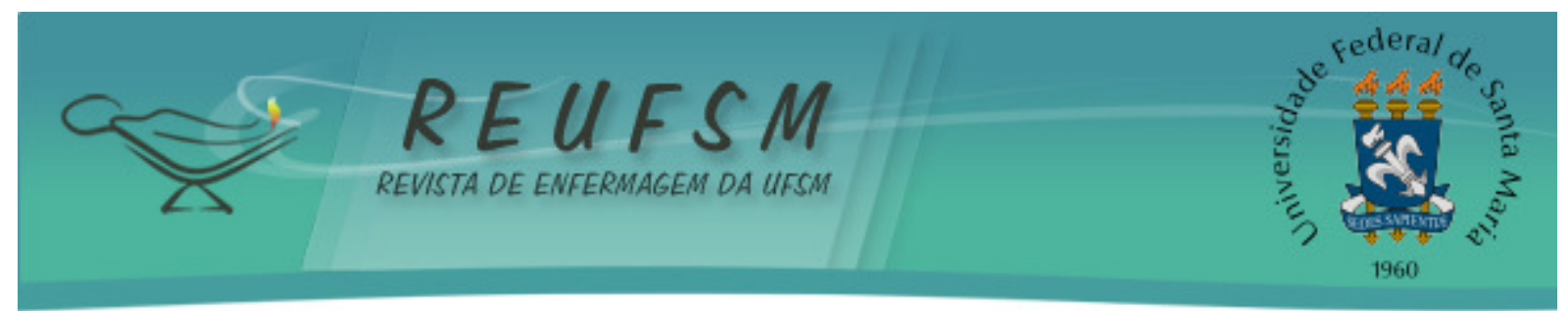

dos horários dos serviços em conformidade a possibilidades dos usuários, estrutura física inadequada e número insuficiente de profissionais de saúde para atender a demanda.

Os aspectos discutidos nesse estudo tornam-se significativos para uma compreensão ampliada dos serviços substitutivos de saúde mental no contexto nacional, à medida que evidencia fragilidades a serem repensadas tanto em âmbito "micro como "macro", contribuindo no aprimoramento de políticas públicas eficientes e adequada as realidades locais, tendo o usuário como protagonista no processo de cuidar em saúde mental.

Como limitação principal da pesquisa tem-se o fato da coleta ter sido realizada em um único serviço da RAPS. No tocante às dificuldades para realização da pesquisa, o acesso aos usuários tornou-se difícil, visto que o tempo para aplicação dos questionários era limitado, tendo em vista o fluxo e demanda de atividades do serviço. Recomenda-se que sejam desenvolvidas mais pesquisas em outros cenários sobre a temática em questão.

\section{REFERÊNCIAS}

1. Miranda L, Oliveira TFK, Santos CBT. Estudo de uma Rede de Atenção Psicossocial: paradoxos e efeitos de precariedade. Psicol Ciênc Prof [Internet]. 2014 [acesso em 2016 ago 19];34(30):592-611. Disponível em: http://www.scielo.br/pdf/pcp/v34n3/1982-3703-pcp-3403-0592.pdf.

2. Barbosa GC, Oliveira M AF, Moreno V, Padovani CR, Claro HG, Pinho PH. Satisfação de usuários em um Centro de Atenção Psicossocial em álcool e outras drogas. Rev Portuguesa de Enferm Saúde Mental [Internet]. 2015 dez [acesso em 2017 jun 2];14:31-7. Disponível em: http://www.scielo.mec.pt/pdf/rpesm/n14/n14a05.pdf.

3. Oliveira VF, Alves JS, Moraes ACS, Silva JC, Silva CSS, Nepomuceno FWAB, et al. Caracterização de pacientes com transtornos mentais atendidos no centro de atenção psicossocial em São Francisco do Conde - Bahia. Rev Ciênc Méd Biol. 2014;13(2):204-11.

4. Kantorski LP, Machado RA, Lemões MAM, Quadros LCM, Coimbra VCC, Jardim VMR. Avaliação da estrutura e processo na visão dos familiares de usuários de saúde mental. Ciênc Cuid Saúde. 2012;11(1):173-80.

5. Minayo, MCS. O desafio do conhecimento: pesquisa qualitativa em saúde. $14^{\mathrm{a}}$ ed. São Paulo: Hucitec; 2014.

6. CAPSUL. Avaliação dos CAPS da Região Sul do Brasil: Relatório. Pelotas: Ministério da Saúde, Conselho Nacional de Desenvolvimento Científico e Tecnológico - CNPq; 2007. p. 437.

7. Bardin L. Análise de conteúdo. São Paulo: Almedina, 2011.

8. Lima AIO, Severo AK, Andrade Nl, Soares GP, Silva LM. O desafio da construção do cuidado integral em saúde mental no âmbito da atenção primária. Temas em Psicologia. 2013; 21(1):71-82. 


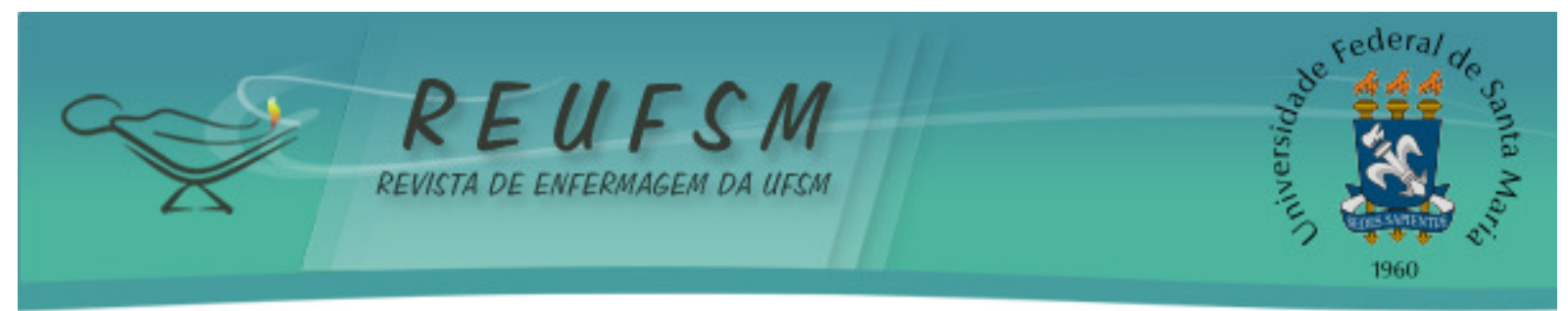

9. Mielke FB, Olshowsky A. Actions of mental health in family health strategy and the health technologies. Esc Anna Nery Rev Enferm. 2011;15(4):762-8.

10. Araújo AK, Tanaka OY. Avaliação do processo de acolhimento em Saúde Mental na região centro-oeste do município de São Paulo: a relação entre CAPS e UBS em análise. Rev Interface Comunic Saúde Educ. 2012;16(43):917-28.

11. Souza LGS, Pinheiro LB. Oficinas terapêuticas em um Centro de Atenção Psicossocial álcool e drogas. Aletheia [Internet]. 2012 [acesso em 2017 jul 15];(38-39):218-27. Disponível em: http://pepsic.bvsalud.org/scielo.php?script=sci_arttext\&pid=S1413-03942012000200018.

12. Noronha AA, Folle D, Guimarães AN, Bruma MLB, Schneider JF, Motta MGC. Percepções de familiares de adolescentes sobre oficinas terapêuticas em um centro de atenção psicossocial infantil. Rev Gaúcha Enferm. 2016;37(4).

13. Oliveira JFM, Silva RJG. Perfil sociodemográfico de pessoas com transtorno mental: um estudo num centro de atenção psicossocial. Rev Eletrônica Gestão \& Saúde [Internet]. 2014 [acesso em 2016 set 29];5(3):862-72. Disponível em: http://periodicos.unb.br/index.php/rgs/article/view/22686.

14. Farias ID, Thofehrn MB, Andrade APM, Carvalho LA, Fernandes HN, Porto AR. Oficina terapêutica como expressão da subjetividade. Rev Eletrônica Saúde Mental Álcool Drog. 2016;12(3):147-53.

15. Oliveira LV, Cirilo LS, Costa GMC. O cuidar do portador de transtorno mental: significado para a família. Rev Baiana Saúde Pública [Internet]. 2013 [acesso em 2016 set 7];37(1):164-78. Disponível

http://inseer.ibict.br/rbsp/index.php/rbsp/article/view/364/364.

16. Oliveira GC de, Nasi C, Lachinni AJB, Camatta MW, Maltz C, Schneider JF. A reabilitação psicossocial: processo de reconstrução da subjetividade do usuário de drogas. Rev Enferm UERJ [Internet]. 2015 [acesso em 2017 fev 15];23(6):811-6. Disponível em: http://www.epublicacoes.uerj.br/index.php/enfermagemuerj/article/view/11742.

17. Paranhos-Passos F, Aires S. Reinserção social de portadores de sofrimento psíquico: o olhar de usuários de um Centro de Atenção Psicossocial. Physis [Internet]. 2013 [acesso em 2017 jun 8]; 23(1): 13-31. Disponível em: http://www.scielo.br/scielo.php?script=sci_arttext\&pid=S0103-73312013000100002.

18. Nóbrega MPSS, Silva GBF, Sena ACR. Funcionamento da Rede de Atenção Psicossocial - RAPS no município de São Paulo, Brasil: perspectivas para o cuidado em saúde mental. Atas CIAIQ [Internet]. 2016 [acesso em 2016 ago 29];2:1-9. Disponível em: http://proceedings.ciaiq.org/index.php/ciaiq2016/article/viewFile/735/722.

19. Paiano M, Maftum MA, Hadda MCL, Marcon SS. Ambulatório de saúde mental: fragilidades apontadas por profissionais. Texto \& Contexto Enferm [Internet]. 2016 [acesso em 2016 set 15];25(3). Disponível em: http://www.scielo.br/pdf/tce/v25n3/pt_0104-0707-tce25-03-0040014.pdf.

20. Azevedo DM, Oliveira AM, Melo GSM, Salvetti MG, Vasconcelos QLDAQ, Torres GV. Avaliação da assistência em saúde num centro de atenção psicossocial na perspectiva dos profissionais. Rev Bras Pesqui Saúde. 2014;16(2):109-16. 


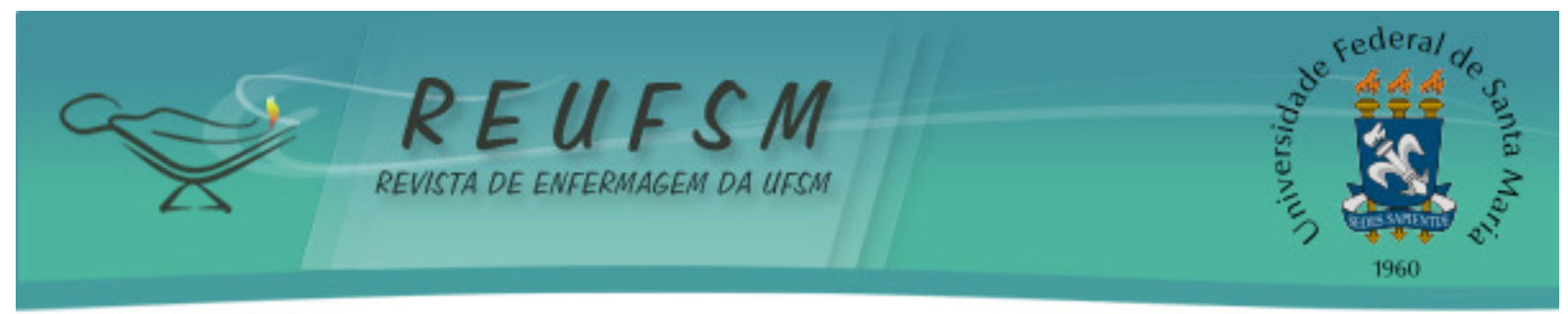

Data de submissão: 21/02/2017

Data de aceite: $21 / 09 / 2017$

Autor correspondente: Francisco de Sales Clementino

Email: fclementino67@yahoo.com.br

Endereço: João Machado, 412, Prata, Campina Grande, Paraíba.

CEP: 58400510 